\title{
Flux Correlations in Supersonic Isothermal Turbulence
}

\author{
R. W A G N E R $\mathbf{R}^{1} \dagger$, G. F A L K O V I C H \\ A. G. KRIT SUK ${ }^{3}$ AND M. L. NORMA N $\mathbf{N}^{1,3}$ \\ ${ }^{1}$ San Diego Supercomputer Center, University of California, San Diego, MC 0505, 10100 \\ Hopkins Drive, La Jolla, CA 92093-0505, USA \\ ${ }^{2}$ Physics of Complex Systems, Weizmann Institute of Science, Rehovot 76100, Israel \\ ${ }^{3}$ Department of Physics and Center for Astrophysics and Space Sciences, University of \\ California, San Diego, MC 0424, 9500 Gilman Drive, La Jolla, CA 92093-0424, USA
}

(Received ?; revised ?; accepted ?. - To be entered by editorial office)

Using data from a large-scale three-dimensional simulation of supersonic isothermal turbulence, we have tested the validity of an exact flux relation derived analytically from the Navier-Stokes equation by Falkovich, Fouxon and Oz [2010 New relations for correlation functions in Navier-Stokes turbulence. J. Fluid Mech. 644, 465]. That relation, for compressible barotropic fluids, was derived assuming turbulence generated by a largescale force. However, compressible turbulence in simulations is usually initialized and maintained by a large-scale acceleration, as in gravity-driven astrophysical flows. We present a new approximate flux relation for isothermal turbulence driven by a large-scale acceleration, and find it in reasonable agreement with the simulation results.

\section{Introduction}

An important rigorously derived result in the statistical theory of incompressible turbulence is Kolmogorov's four-fifths law (Kolmogorov 1941)

$$
\left\langle\left[\delta u_{\|}(r)\right]^{3}\right\rangle=-\frac{4}{5} \epsilon r,
$$

where

$$
\delta u_{\|}(r) \equiv[\boldsymbol{u}(\boldsymbol{r})-\boldsymbol{u}(0)] \cdot \boldsymbol{r} / r
$$

is the longitudinal velocity difference between two points, 0 and $\boldsymbol{r}$, separated by a distance $r=|\boldsymbol{r}|$, and $\epsilon$ is the mean energy dissipation rate. The four-fifths law is well supported experimentally and has been traditionally interpreted as a signature of a direct kinetic energy cascade within the inertial range of scales. Recent work by Falkovich et al. (2010, hereafter FFO), reinterprets the Kolmogorov relation in terms of currents and densities of the conserved quantities. From this new perspective, FFO derived an analytic scaling relation for barotropic flows with an arbitrary degree of compressibility, based on the correlation of currents and conserved fluxes in the inertial range.

There are few analytic relations proposed for compressible turbulence, e.g., the relation for an isothermal gas based on an effective energy transfer rate Galtier \& Banerjee 2011), and the lack of experimental results at high turbulent Mach numbers makes verification of such relations challenging. While supersonic turbulence is observed in molecular clouds (Heyer \& Brunt 2004), and is believed to play an important role in star

$†$ Email address for correspondence: rpwagner@sdsc.edu 
formation (McKee \& Ostriker 2007), observations are limited by available angular resolution, projection and finite optical depth effects (Elmegreen \& Scalo 2004). At present, numerical experiments provide the best route for testing theories of compressible turbulence (Svtine et al. 2000; Benzi et al. 2008; Kitsionas et al. 2009; Kritsuk et al. 2011; Brandenburg \& Nordlund 2011). Three-dimensional numerical simulations show that even at very high Mach numbers, a compressible energy cascade can be recovered with a proper density weighting, $\rho^{1 / 3} \boldsymbol{u}$ (Kritsuk et al. 2007a, b; Pan et al. 2009). Aluie et al. (2012) have recently presented evidence from simulations for pressure-dilatation action at large scales, and kinetic energy transfer to the dissipation scale via a conservative cascade, supporting an earlier analytical proof of the locality of kinetic energy transfer in compressible turbulence (Aluie 2011).

In this article, we use data from a simulation of supersonic isothermal turbulence to analyze the exact relation for compressible fluids from FFO. We are particularly interested in evaluating the role of the properties of the force that acts as a source of momentum and energy to compensate for the dissipation losses. We discuss the limitations implied by the assumptions used in the FFO derivation and present a new approximate scaling relation appropriate for the driving commonly used in numerical experiments on compressible fluid turbulence (e.g., Porter et al. 2002; Kritsuk et al. 2007a; Schmidt et al. 2008; Wang et al. 2010).

\section{Flux Correlations}

A general relation is derived in FFO, along with a particular case for compressible turbulence in a barotropic fluid, both of which are based on two-point statistics in the inertial range. The lower limit of $r$ where the relations are expected to hold is the scale where dissipation is negligible, while the upper limit is the correlation length of the force. The correlations involve the densities $q^{a}$, currents $\boldsymbol{j}^{a}$, fluxes $F_{i}^{a}$, and sources (forcing) $f^{a}$; the sources are assumed to be random, statistically stationary, spatially homogeneous, and isotropic. Beginning with the governing equations, and eliminating terms tied to dissipation, a relation involving the densities, fluxes, and forcing is derived:

$$
\nabla_{i}\left\langle q^{a}(0, t) F_{i}^{a}(\boldsymbol{r}, t)\right\rangle=\left\langle q^{a}(0, t) f^{a}(\boldsymbol{r}, t)\right\rangle,
$$

see FFO for more detail.

In order to eliminate the scale dependence on the right-hand side of (2.1), the authors assume that on scales much smaller than the correlation length $(r \ll L)$, the force is approximately constant,

$$
f^{a}(0, t) \approx f^{a}(\boldsymbol{r}, t) .
$$

Therefore, in the inertial range, the correlation of a density and its source is also approximately constant,

$$
\left\langle q^{a}(0, t) f^{a}(\boldsymbol{r}, t)\right\rangle \approx\left\langle q^{a}(0, t) f^{a}(0, t)\right\rangle \equiv \bar{\epsilon}_{a} .
$$

Note that this ansatz is essentially carried over from the incompressible case, where there is no distinction between the large-scale acceleration and large-scale force since the 
density is constant $甘$ Substituting $\bar{\epsilon}_{a}$ into (2.1) gives the correlation function

$$
\nabla_{i}\left\langle q^{a}(0) F_{i}^{a}(\boldsymbol{r})\right\rangle=\bar{\epsilon}_{a},
$$

and assuming isotropy, FFO derive the exact scaling relation in a vector form:

$$
\left\langle q^{a}(0) F_{i}^{a}(\boldsymbol{r})\right\rangle=\frac{\bar{\epsilon}_{a} r_{i}}{d},
$$

where $d$ is the number of spatial dimensions.

In the case of a barotropic fluid, the densities are $\boldsymbol{q}=(\rho \boldsymbol{u}, \rho)$, and the fluxes are $F_{i}^{j}=\rho u_{i} u_{j}+p(\rho) \delta_{i j}$ for $i, j=1, \ldots, d$, while $F_{i}^{d+1}=\rho u_{i}$. When dissipation is neglected, the governing equations are the forced Euler equations:

$$
\begin{aligned}
\partial_{t} \rho+\nabla \cdot(\rho \boldsymbol{u}) & =0, \\
\partial_{t}\left(\rho u_{i}\right)+\partial_{j}\left(\rho u_{i} u_{j}+p \delta_{i j}\right) & =f^{i} .
\end{aligned}
$$

Substituting the densities, fluxes, and forcing into (2.5) gives a relation for isothermal turbulence when $p=\rho$,

$$
\Phi(r) \equiv\left\langle[\rho(0) \boldsymbol{u}(0) \cdot \boldsymbol{u}(\boldsymbol{r}) \rho(\boldsymbol{r})] u_{\|}(\boldsymbol{r})\right\rangle+\left\langle\rho(0) u_{\|}(0) \rho(\boldsymbol{r})\right\rangle=\frac{\bar{\epsilon} r}{d},
$$

where $u_{\|}=\boldsymbol{u} \cdot \boldsymbol{r} / r$ is the longitudinal velocity,

$$
\bar{\epsilon}=\langle\rho(0) \boldsymbol{u}(0) \cdot \boldsymbol{f}(0)\rangle,
$$

and we have taken the scalar product of both sides of (2.5) with $\boldsymbol{r} / r$ to get (2.8) in a more convenient scalar form. Unlike $\epsilon$ in (1.1), $\bar{\epsilon}$ is the injection rate of momentum squared, not energy. However, in the incompressible limit, the vector form of (2.8) reduces to a third-order velocity correlation function implying (1.1).

In numerical experiments of compressible turbulence, the flow is traditionally driven by a large-scale acceleration $\boldsymbol{a}$, such that $\boldsymbol{f}=\rho \boldsymbol{a}$, while the exact flux relation (2.8) was derived under the assumption that $f$ is a smooth, large-scale, field. As noted in FFO, physically, $f$ may come from a gradient of potential (e.g., external gravitational acceleration, i.e. $\boldsymbol{f}=\rho \nabla \phi$ or $\boldsymbol{a}=\nabla \phi$ ). The decorrelation and small-scale variations of the density at high Mach number make this an important point, as acceleration-driven turbulence is important in astrophysics and other areas, such as turbulent convection.

Let us suggest a new flux relation appropriate for this case. Now, instead of the constant $\bar{\epsilon}$ in (2.9) we shall have the two-point fourth-order correlation function $\langle\rho(0) \rho(\boldsymbol{r}) \boldsymbol{u}(0)$. $\boldsymbol{a}(\boldsymbol{r})\rangle$, which is scale-dependent. The character of this scale-dependence can be found in the particular case of an acceleration short-correlated in time, when $\left\langle a_{i}(t) a_{j}(0)\right\rangle=\varepsilon_{i j} \delta(t)$. The average of the product of any quantity $U\{\boldsymbol{a}\}$ and a white noise $\boldsymbol{a}$ is expressed by the formula of Gaussian integration via a variational derivative: $\left\langle U\{\boldsymbol{a}\} a_{i}\right\rangle=\varepsilon_{i j}\left\langle\delta U / \delta a_{j}\right\rangle$. In the correlation function we consider, it is the velocity field which is related to the acceleration (by the equation $d u_{i} / d t-u_{i} \nabla \cdot \boldsymbol{u}=a_{i}$ ) so that $\left\langle\delta u_{i}(t) / \delta a_{j}\left(t^{\prime}\right)\right\rangle=\delta_{i j} \theta\left(t-t^{\prime}\right)$ where the last factor is the step function. We now obtain

$$
\langle\rho(0) \rho(\boldsymbol{r}) \boldsymbol{u}(0) \cdot \boldsymbol{a}(\boldsymbol{r})\rangle=\langle\rho(0) \rho(\boldsymbol{r})\rangle \bar{\varepsilon}
$$

where $\bar{\varepsilon}=\langle\boldsymbol{u}(0) \cdot \boldsymbol{a}(0)\rangle$. Since the acceleration is a large-scale field, then a two-point

$\dagger$ While we recognize that the assumption of large-scale force per unit volume is possibly the only viable path to a rigorously derived relation (Galtier \& Baneriee 2011, also relied on the same constraint to come up with an alternative relation for a compressible case), we emphasize that driving variable-density flows with a large-scale force would have a nontrivial effect on the turbulence statistics and eliminate the inertial range in the traditional sense, with respect to the fluid velocity. 
velocity-acceleration correlation function can be replaced by a single-point one. Let us stress that the constant $\bar{\varepsilon}$ is neither the total energy input nor the work of the external acceleration.

Of course, in the physical situations of interest, as well as in simulations, the acceleration cannot be considered short-correlated in time. In this case, the effective decoupling of the fourth moment into the product of second moments expressed by (2.10) is not an exact relation but can be suggested as a plausible approximation. We thus come to the generalization of the flux relation in the following form (up to an order-unity constant):

$$
\nabla_{r} \Phi(r) \simeq\langle\rho(0) \rho(\boldsymbol{r})\rangle \bar{\varepsilon} .
$$

For isothermal turbulence driven by a large-scale acceleration, 2.11) provides an approximate relation connecting the scaling of $\Phi(r)$ with that of $\langle\rho(0) \rho(\boldsymbol{r})\rangle$ in the intertial range.

\section{Experimental Verification}

To appraise the relations from FFO, we have used data from a simulation designed to study the inertial range statistics of highly compressible turbulence (Kritsuk et al. $2007 a$ ). The simulation was performed with an implementation of the piecewise parabolic method (Colella \& Woodward 1984) in the Enzo code (Norman et al. 2007). The forced Euler equations for an isothermal gas, (2.6) and (2.7), were solved numerically in a cubic periodic domain with a linear size of $L=1$ along each axis, and covered by a uniform Cartesian grid of $1024^{3}$ zones, each having sides of length $\Delta=L / 1024$. The turbulent rms Mach number, $M=6$, and a steady rate of energy injection were maintained by a random acceleration field with power limited to wavenumbers $k / k_{\min } \in[1,2]$, where $k_{\text {min }}=2 \pi / L$. The spatially fixed acceleration field was normalized at every time step so that $\langle\rho \boldsymbol{u} \cdot \boldsymbol{a}\rangle$ was constant; the normalization factor had a standard deviation of $\sim 5 \%$ during the simulation. For this work, we used 43 full data snapshots evenly distributed in time in the range $t / \tau \in[6,10]$, where the flow crossing (large eddy turnover) time, $\tau \equiv L / 2 M$, assumes the sound speed of unity. For each snapshot, we computed $\bar{\epsilon}, \bar{\varepsilon}$, and evaluated $\Phi(r)$ for 16 discrete $r$-values in the range $r / \Delta \in[8,128]$, using $2^{32} \approx 4 \times 10^{9}$ random point pairs for each value of $r$.

To evaluate the role of various assumptions concerning the forcing and assess the hypotheses employed by FFO, we compare $\Phi$ based on the exact forms (2.4) and (2.8) with an approximate expression based on (2.11). We begin by examining the spatial distribution of the force and acceleration in the lower right-hand panels of figure 1 which show the values of the $x$ component of $\boldsymbol{a}$ and $\boldsymbol{f}^{\prime}$, in a slice through the simulation volume at $x=0$, where $\boldsymbol{f}^{\prime} \equiv \rho / \bar{\rho} \boldsymbol{a}$ is the force normalized by the average density. A more quantitative assessment can be found in figure 2, which shows the power spectra of both $\boldsymbol{a}$ and $\boldsymbol{f}^{\prime}$. The power spectrum of $\boldsymbol{a}$ falls off at $k / k_{\min }>2$, while the spectrum of $\boldsymbol{f}^{\prime}$ slowly decreases with $k$, and the two are separated by several orders of magnitude above $k / k_{\min } \approx 2$. Further, we measure $\bar{\epsilon}=589, \bar{\varepsilon}=149,\left\langle\rho^{2}\right\rangle=3.74$, and find that

$$
\left\langle\rho^{2}\right\rangle \bar{\varepsilon}=0.95 \bar{\epsilon}
$$

supporting the decoupling suggested in (2.10). This decoupling, combined with the features of $\boldsymbol{f}$ shown by figures 1 and 2 indicates that the assumption of an approximately constant force does not hold in the simulation, as expected.

Next, we will compare $\Phi$ to the scaling from (2.11). We find that the density correlation 

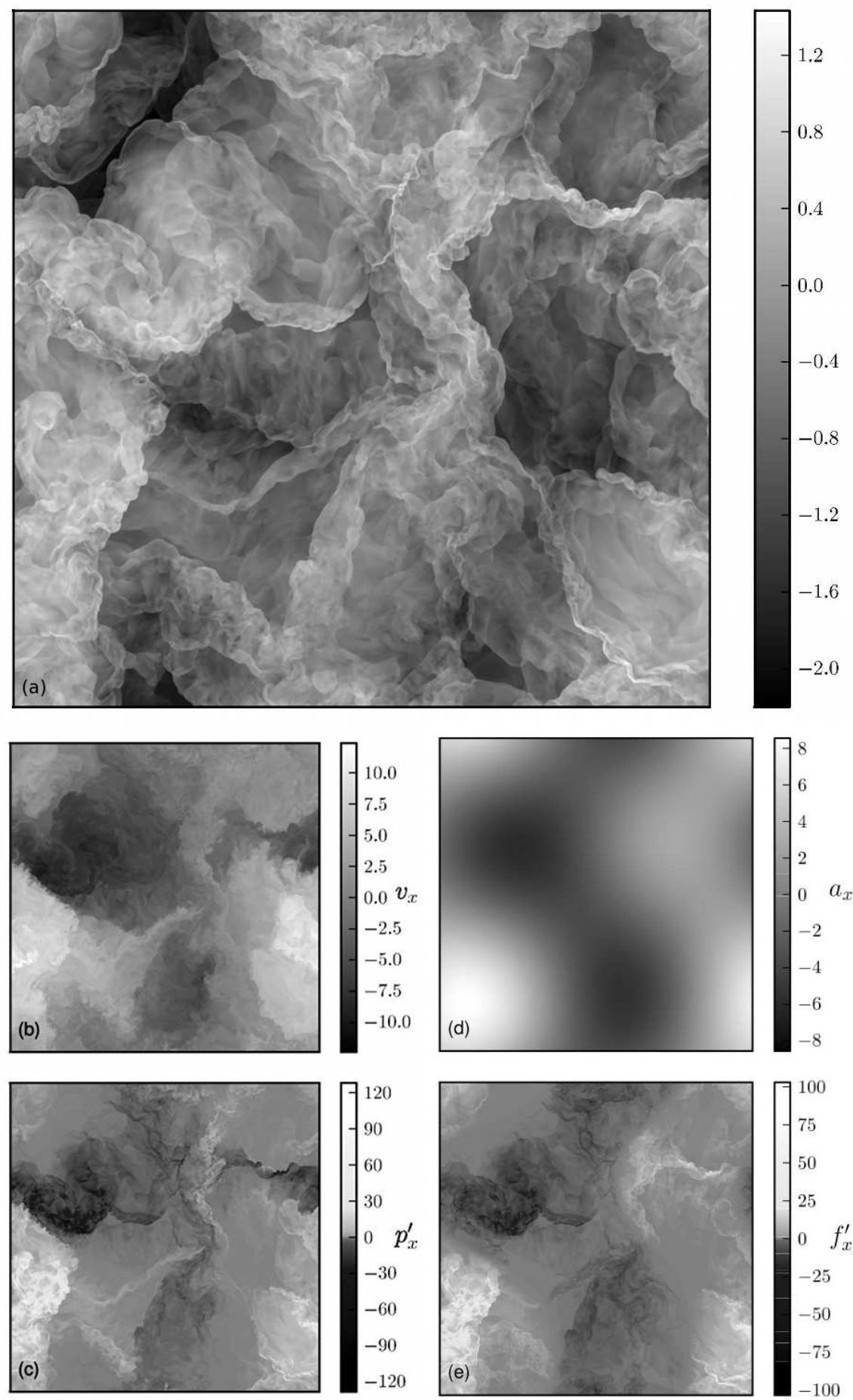

FiguRE 1. Slices of several instantaneous dynamical fields through the plane $x=0$ : (a) logarithm of the normalized density field $\log _{10}(\rho / \bar{\rho}) ;(\mathrm{b})$ velocity field $u_{x}$ (velocity is normal to the image plane); (c) normalized momentum $p_{x}^{\prime}=\rho / \bar{\rho} u_{x}$; (d) acceleration field $a_{x}$; (e) normalized force $f_{x}^{\prime}=\rho / \bar{\rho} a_{x}$. N.B.: To account for the distribution of the density field, the colour maps used for $p_{x}^{\prime}$ and $f_{x}^{\prime}$ are highly compressed around 0 . 


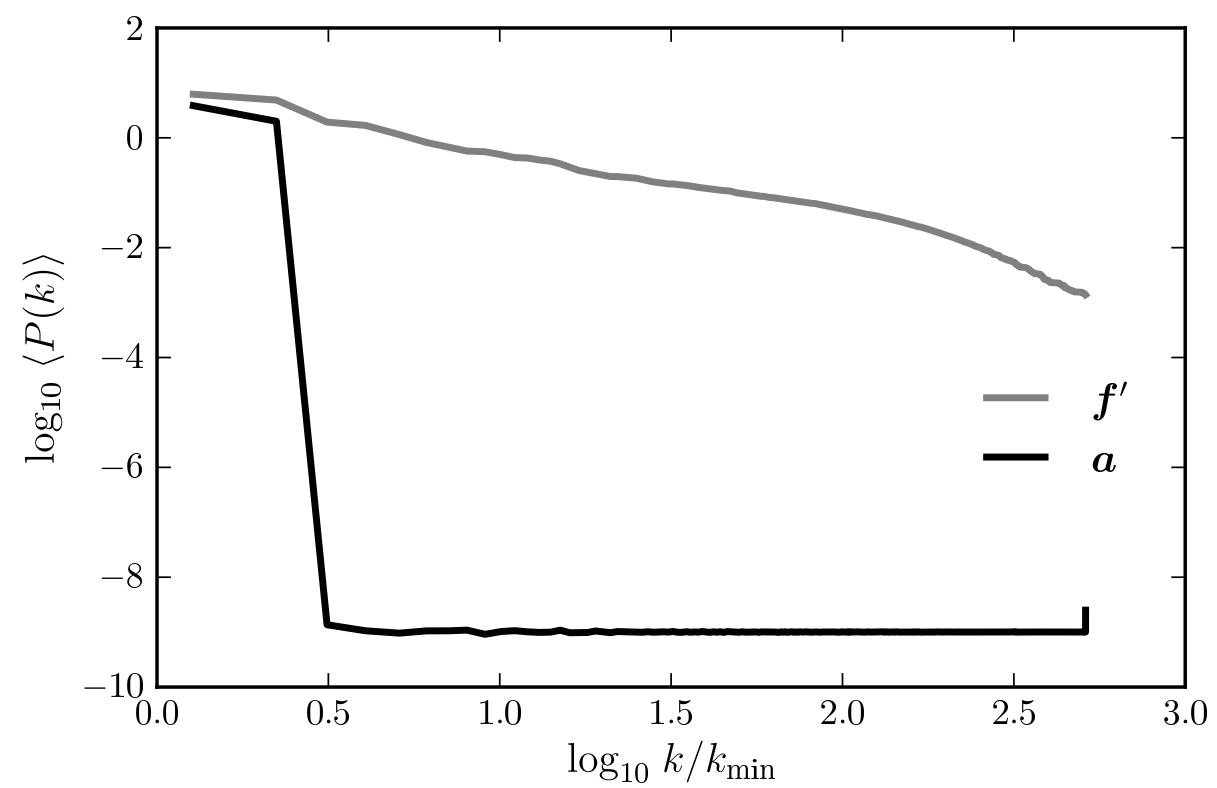

Figure 2. Power spectra of the driving acceleration, $\boldsymbol{a}$ (solid black line), and the normalized force, $\boldsymbol{f}^{\prime}=\rho \boldsymbol{a} / \bar{\rho}$ (solid grey line), from a single flow snapshot.

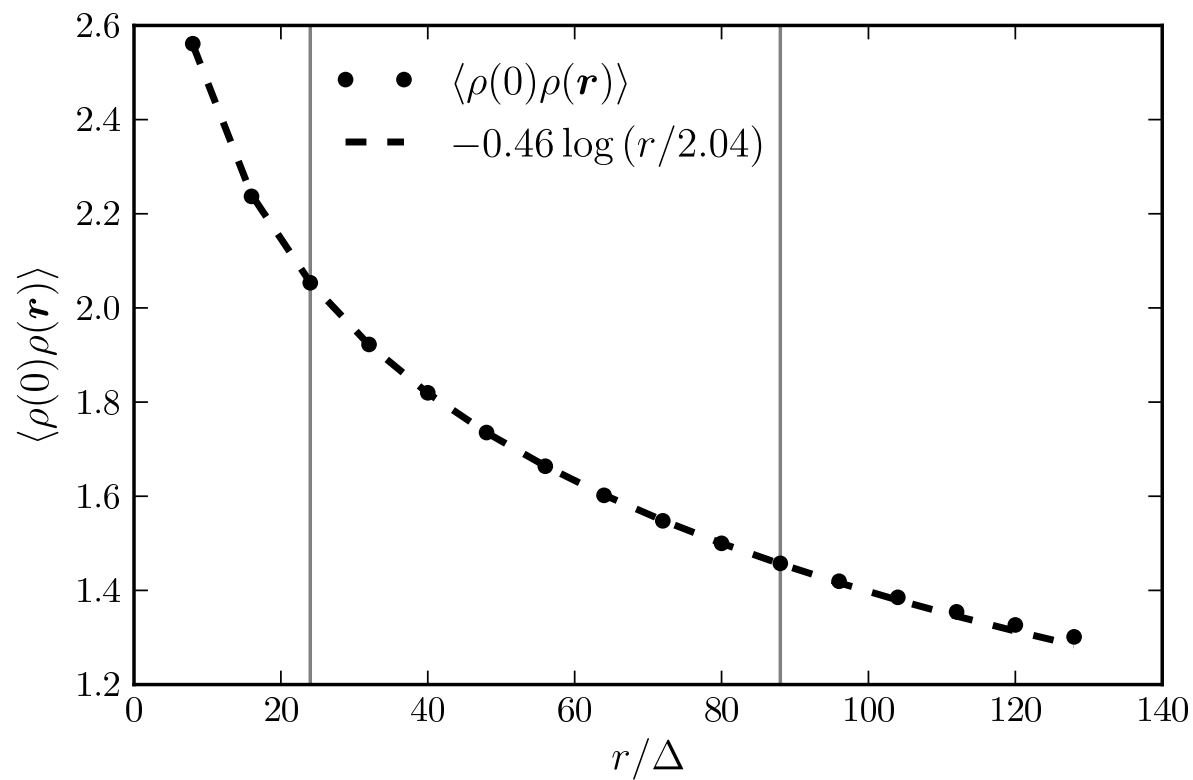

Figure 3. Dots: time-averaged density correlation; the standard error of the mean of $\langle\rho(0) \rho(\boldsymbol{r})\rangle$ is $<0.02$. Dashed line: best fit to the data points with $a \log (r / b)$ in the range $r / \Delta \in[20,90]$, as indicated by the vertical grey lines. 


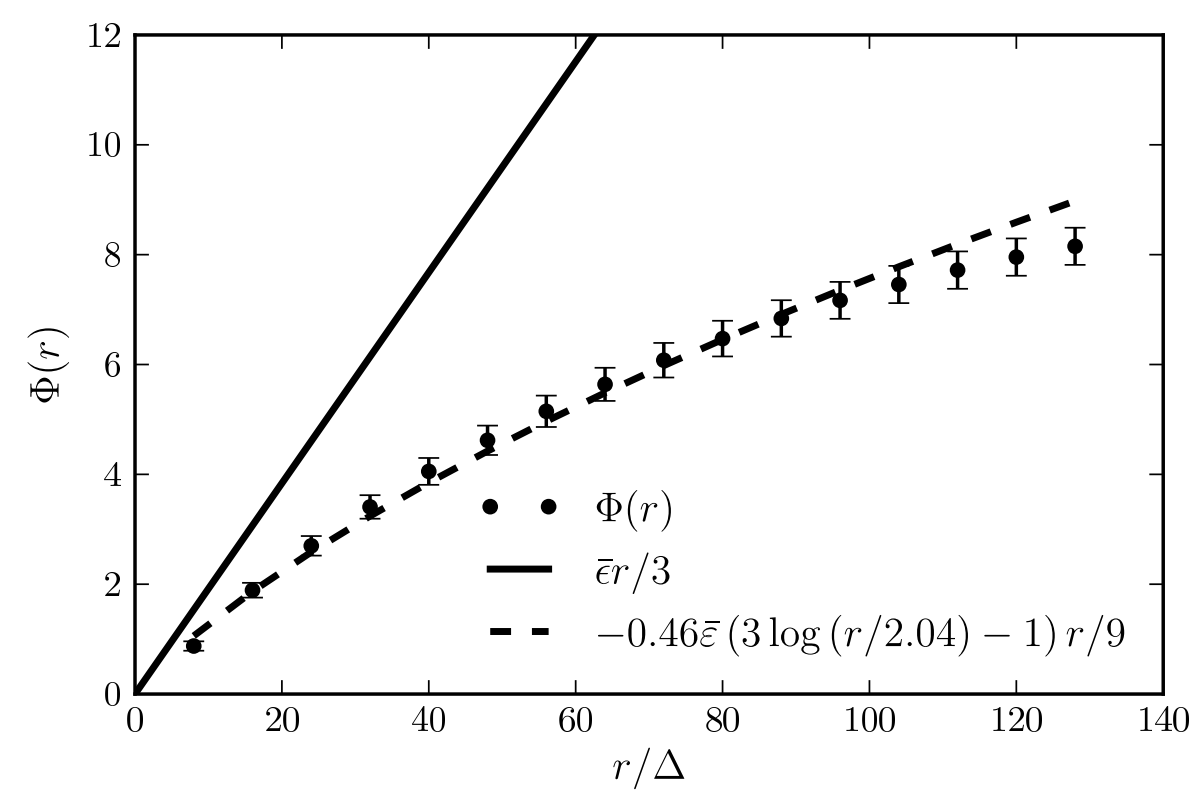

FiguRE 4. Dots: time-averaged measure of $\Phi(r)$; the error bars show the standard error of the mean. Solid line: expected scaling based on a large-scale force. Dashed line: expected approximate scaling based on the density correlation. Note that the dashed line is not a fit to the $\Phi(r)$ points, but shows the right-hand side of (3.3) with parameters determined by the best fit to the density correlation (3.2) shown in figure 3 .

is well approximated by a logarithmic form:

$$
\langle\rho(0) \rho(\boldsymbol{r})\rangle \simeq a \log \left(\frac{r}{b}\right)
$$

with $a=-0.46 \pm 0.001$ and $b=2.04 \pm 0.01$ when fitted in the range $r / \Delta \in[20,90]$ (see figure 3). This is expected from the density power spectrum approximately following $\sim k^{-1}$ at this particular turbulent Mach number (Kritsuk et al. 2007a). Substituting (3.2) into (2.11) and integrating radially from 0 to $r$ assuming isotropy, we have

$$
\Phi(r) \simeq \frac{a \bar{\varepsilon}}{9}\left[3 \log \left(\frac{r}{b}\right)-1\right] r .
$$

Note that (3.3) is a good approximation only for turbulence at $M=6$. Simulations with different Mach numbers will have density power spectra with different slopes, and their density correlations will have different forms.

Figure 4 s shows the time-averaged $\Phi(r)$, the expected scaling for a large-scale force using (2.8), and the expected scaling based on a large-scale acceleration and our approximation (3.3). The accuracy of the approximate flux relation can be assessed in figure 5 where we show $\Phi(r)$ normalized by the right-hand side of (3.3), using the values fitted to the density correlation. The right-hand side of (3.3) follows the actual measured $\Phi(r)$ within $\pm 5 \%$ in the range $r / \Delta \in[10,100]$, somewhat underestimating $\Phi$ at the lower end of the range, and overestimating it as $r$ increases; this behaviour can be predicted as the assumed approximations break down for two different reasons. First, from (3.1), the density correlation and injection rate of specific kinetic energy are not totally decoupled; this may account for (2.11) underestimating $\Phi$ at small scales. Second, as $r$ approaches 


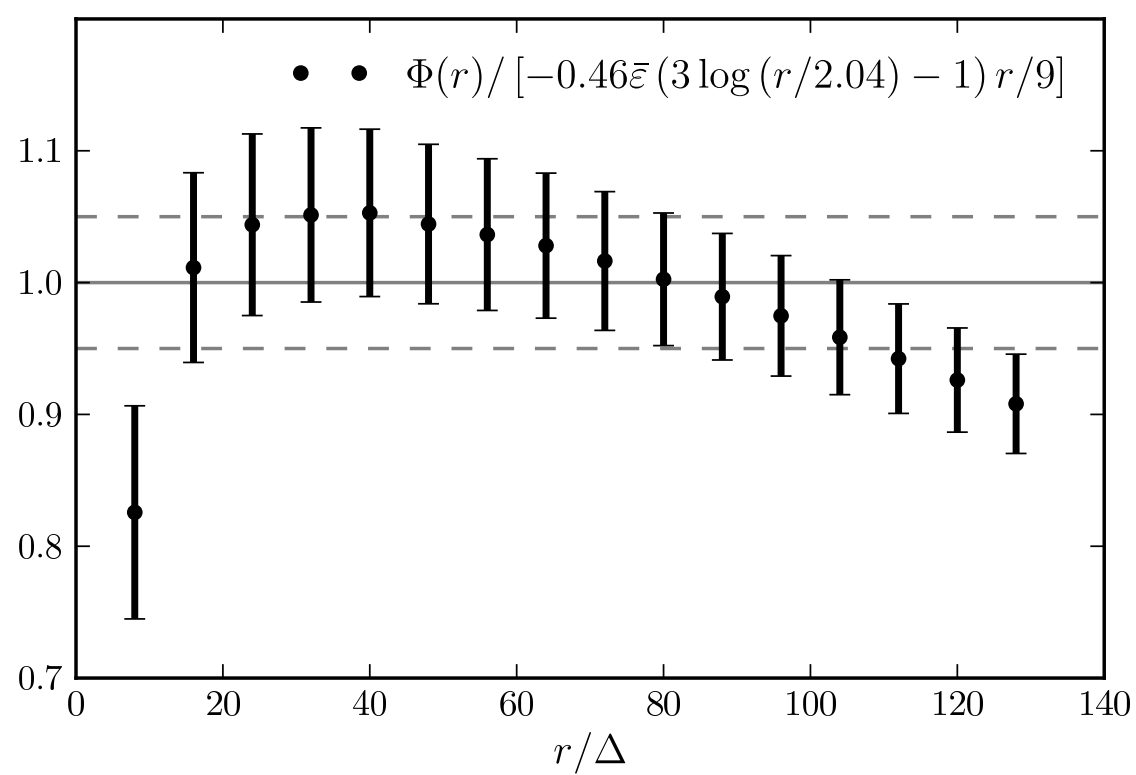

Figure 5. Time-averaged measure of $\Phi(r)$ normalized by the expected approximate scaling (3.3). The dashed grey lines show $\pm 5 \%$ tolerance levels.

$L / 2,\langle\boldsymbol{u}(0) \cdot \boldsymbol{a}(\boldsymbol{r})\rangle \rightarrow 0$, and as (2.2) breaks down, a constant $\bar{\varepsilon}$ on the right-hand side of (2.11) causes it to excede $\Phi(r)$. This departure by $\Phi(r)$ from (3.3) at large scales is determined by the numerical resolution and driving scales; given a similar simulation with higher numerical resolution, we would expect the agreement to scale relative to the smallest driving scale.

\section{Conclusion}

We find that the approximate relation we derived for supersonic isothermal turbulence driven by a large-scale acceleration field (2.11) agrees reasonably well with the simulation results within a sufficiently wide range of scales consistent with the inertial range presence established in Kritsuk et al. (2007a). This implies support for the original FFO exact relation (2.1), so it can be anticipated that in a system where (2.3) holds, the exact relation (2.8) will be satisfied as well. Therefore, figures 4 and 5 demonstrate the strong sensitivity of the flux correlations in supersonic isothermal turbulence to the nature of forcing supporting the statistically stationary state. Such sensitivity has been observed in a similar context of the forced one-dimensional Burgers equation, where an invasive random force with a $\propto k^{-1}$ spectrum (compare with the grey line in figure 2) would produce a non-universal bifractal scaling (Mitra et al. 2005). Likewise, Bahraminasab et al. (2008) showed that the same Burgers system driven by a force with large-scale correlation in space and with a Wiener scaling in time exhibits a non-universal scaling for time increments larger than the correlation time of the force. Comparison with other relations and results addressing energy transfer in compressible turbulence (Galtier \& Banerjee 2011; Aluie 2011; Aluie et al. 2012) will help us to understand whether non-universality, observed here due to the driving mechanism, is a more general property of highly compressible flows. 


\section{Acknowledgements}

R.W. is supported in part by CyberInfrastructure Research, Education and Development at the San Diego Supercomputer Center (SDSC); A.K. is supported in part by NSF grants AST-0908740 and AST-1109570. The simulation utilized TeraGrid computer time allocations MCA98N020 and MCA07S014 at SDSC. The analysis was performed on the Extreme Science and Engineering Discovery Environment (XSEDE) resource Trestles at SDSC under a Director's Discretionary Allocation. A.K. and G.F. were supported in part by the Project of Knowledge Innovation Program (PKIP) of Chinese Academy of Sciences, Grant No. KJCX2.YW.W10.

\section{REFERENCES}

Aluie, H. 2011 Compressible turbulence: The cascade and its locality. Phys. Rev. Lett. 106 (17), 174502.

Aluie, H., Li, S. \& LI, H. 2012 Conservative Cascade of Kinetic Energy in Compressible Turbulence. Astrophys. J., Lett. 751, L29.

Bahraminasab, A., Niry, M. D., Davoudi, J., Reza Rahimi Tabar, M., Masoudi, A. A. \& Sreenivasan, K. R. 2008 Taylor's frozen-flow hypothesis in burgers turbulence. Phys. Rev. E 77, 065302.

Benzi, R., Biferale, L., Fisher, R. T., Kadanoff, L. P., Lamb, D. Q. \& Toschi, F. 2008 Intermittency and Universality in Fully Developed Inviscid and Weakly Compressible Turbulent Flows. Phys. Rev. Lett. 100 (23), 234503.

Brandenburg, A. \& Nordlund, A. 2011 Astrophysical turbulence modeling. Rep. Progr. Phys. 74 (4), 046901.

Colella, P. \& Woodward, P. R. 1984 The Piecewise Parabolic Method (PPM) for GasDynamical Simulations. J. Comp. Phys. 54, 174-201.

Elmegreen, B. G. \& Scalo, J. 2004 Interstellar Turbulence I: Observations and Processes. Annual Review of Astron and Astrophys 42, 211-273.

Falkovich, G., Fouxon, I. \& Oz, Y. 2010 New relations for correlation functions in NavierStokes turbulence. J. Fluid Mech. 644, 465-472.

Galtier, S. \& BanerJee, S. 2011 Exact Relation for Correlation Functions in Compressible Isothermal Turbulence. Phys. Rev. Lett. 107 (13), 134501.

Heyer, M. H. \& Brunt, C. M. 2004 The Universality of Turbulence in Galactic Molecular Clouds. Astrophys. J., Lett. 615, L45-L48.

Kitsionas, S., Federrath, C., Klessen, R. S., Schmidt, W., Price, D. J., Dursi, L. J., Gritschneder, M., Walch, S., Piontek, R., Kim, J., Jappsen, A.-K., Ciecielag, P. \& Mac Low, M.-M. 2009 Algorithmic comparisons of decaying, isothermal, supersonic turbulence. A\&A 508, 541-560.

Kolmogorov, A. N. 1941 Dissipation of Energy in the Locally Isotropic Turbulence. Dokl. Akad. Nauk SSSR 32, 19.

Kritsuk, A. G., Nordlund, A., Collins, D., Padoan, P., Norman, M. L., Abel, T., Banerjee, R., Federrath, C., Flock, M., Lee, D., Li, P. S., Müller, W.-C., Teyssier, R., Ustyugov, S. D., Vogel, C. \& Xu, H. 2011 Comparing Numerical Methods for Isothermal Magnetized Supersonic Turbulence. Astrophys. J. 737, 13.

Kritsuk, A. G., Norman, M. L., Padoan, P. \& Wagner, R. $2007 a$ The Statistics of Supersonic Isothermal Turbulence. Astrophys. J. 665, 416-431.

Kritsuk, A. G., Padoan, P., Wagner, R. \& Norman, M. L. $2007 b$ Scaling laws and intermittency in highly compressible turbulence. In Turbulence and Nonlinear Processes in Astrophysical Plasmas (ed. D. Shaikh \& G. P. Zank), American Institute of Physics Conference Series, vol. 932, pp. 393-399.

McKee, C. F. \& Ostriker, E. C. 2007 Theory of Star Formation. Annu. Rev. Astron. Astr. 45, 565-687.

Mitra, D., Bec, J., Pandit, R. \& Frisch, U. 2005 Is Multiscaling an Artifact in the Stochastically Forced Burgers Equation? Physical Review Letters 94 (19), 194501.

Norman, M. L., Bryan, G. L., Harkness, R., Bordner, J., Reynolds, D., O’Shea, B. 
\& Wagner, R. 2007 Simulating Cosmological Evolution with Enzo, chap. 5, pp. 83-102. CRC Press.

Pan, L., Padoan, P. \& Kritsuk, A. G. 2009 Dissipative Structures in Supersonic Turbulence. Phys. Rev. Lett. 102 (3), 034501.

Porter, D., Pouquet, A. \& Woodward, P. 2002 Measures of intermittency in driven supersonic flows. Phys. Rev. E 66 (2), 026301.

Schmidt, W., Federrath, C. \& Klessen, R. 2008 Is the Scaling of Supersonic Turbulence Universal? Phys. Rev. Lett. 101 (19), 194505.

Sytine, I. V., Porter, D. H., Woodward, P. R., Hodson, S. W. \& Winkler, K.-H. 2000 Convergence Tests for the Piecewise Parabolic Method and Navier-Stokes Solutions for Homogeneous Compressible Turbulence. J. Comp. Phys. 158, 225-238.

Wang, J., Wang, L.-P., Xiao, Z., Shi, Y. \& Chen, S. 2010 A hybrid numerical simulation of isotropic compressible turbulence. J. Comp. Phys. 229, 5257-5279. 